\title{
Patients with hMLH1 or/and hMSH2-deficient Metastatic Colorectal Cancer Are Associated with Reduced Levels of Vascular Endothelial Growth Factor-1 Expression and Higher Response Rate to Irinotecan-based Regimen
}

\author{
RIYAD BENDARDAF ${ }^{1,2}$, FATEMEH SAHEB SHARIF-ASKARI ${ }^{3}$, \\ NARJES SAHEB SHARIF-ASKARI ${ }^{3}$, KARI SYRJÄNEN ${ }^{4}$ and SEPPO PYRHÖNEN ${ }^{5}$ \\ ${ }^{1}$ Oncology unit, University Hospital Sharjah, Sharjah, United Arab Emirates; \\ ${ }^{2}$ Department of Clinical Sciences, College of Medicine, University of Sharjah, Sharjah, United Arab Emirates; \\ ${ }^{3}$ Sharjah Institute of Medical Research, College of Medicine, University of Sharjah, Sharjah, United Arab Emirates; \\ ${ }^{4}$ Department of Clinical Research, Biohit Oyj, Helsinki, Finland; \\ ${ }^{5}$ Department of Oncology and Radiotherapy, Turku University Hospital and University of Turku, Turku, Finland
}

\begin{abstract}
Background/Aim: The benefit of IFL (irinotecan, fluorouracil and leucovorin) regimen for metastatic colorectal cancer patients (mCRCs) with high levels of microsatellite instability (MSI-H) or loss of mismatch repair (dMMR) protein expression, is uncertain. This study investigated the association of tumour MMR-status and VEGF-1 expression with response to first-line IFL regimen in $m C R C s$. Patients and Methods: This prospective study analyzed patients diagnosed with mCRC between August 1st, 1998, and August 30th, 2003, at the Turku University Hospital, Finland. All patients received postoperative IFL regimen. Tumour expression of the MMR proteins, hMLH1 and hMSH2, and VEGF-1 expression were assessed by immunohistochemistry (IHC). Tumours with dMMR were those demonstrating loss of $M M R$ protein expression, and tumours with high VEGF-1 expression were those showing moderate or strong cytoplasmic staining. The primary endpoint was the association between tumour hMLH1 or/and hMSH2-deficient and VEGF-1 expression; the relation between tumour MMRstatus and IFL response rate was the secondary endpoint. Results: Of the $67 \mathrm{mCRCs}$ patients, 29 (43\%) were hMLH1 or/and hMSH2-deficient and 15 (22\%) were pMMR mCRCs.
\end{abstract}

This article is freely accessible online.

Correspondence to: Fatemeh Saheb Sharif-Askari, Sharjah Institute of Medical Research, College of Medicine, University of Sharjah, Sharjah, United Arab Emirates. Tel: +971 501829166, e-mail: fsharifaskari@sharjah.ac.ae

Key Words: Metastatic colorectal cancer, MMR, MSH2, MLH1, VEGF-1, irinotecan.
At diagnosis, patients with hMLH1 or/and hMSH2-deficient tumours expressed lower levels of VEGF-1 compared to pMMR tumour patients $(p=0.01)$. More than half $(n=17,59 \%)$ of those with dMMR were chemosensitive to first-line IFL regimen, while just one-fifth $(n=3,20 \%)$ of those with $p M M R$ were chemosensitive to the IFL regimen $(p=0.045)$. Conclusion: Association between MMR-status and VEGF-1 expression predicts clinical outcome in $m C R C$ patients.

Colorectal cancer (CRC), the third most common cancer worldwide (1), it develops through accumulated genetic changes resulting in loss of expression of tumour suppressor genes and activation of oncogenes. In sporadic CRCs, these genetic changes occur through two different mechanisms. First, chromosomal instability (CIN), occurs in $85 \%$ of sporadic CRC, is due to loss of heterozygosity at multiple tumour suppressor loci (2). Second, microsatellite instability (MSI)/ or deficient mismatch repair (dMMR), occurs in $15 \%$ of sporadic CRCs, and is due to germline mutations in DNA mismatch repair genes ( $M L H 1, M S H 2, M S H 6$, or PMS2) or somatic epigenetic silencing of $M L H 1$ (3).

Identification of MSI can be done either by a genetic approach, by analysing DNA extracted from tumour and normal tissues, or, by immunohistochemistry (IHC), which detects the expression of MLH1 and MSH2, two MMR proteins most commonly lost in sporadic CRC. IHC is a commonly used test used clinically to differentiate MMR deficient (dMMR) from mismatch repair proficient (pMMR) tumours (4).

The standard first-line chemotherapy for CRCs includes 5-fluorouracil with leucovorin and irinotecan $(5,6)$ or oxaliplatin (7) alone or combined with an anti-vascular endothelial growth factor (VEGF) agent, such as bevacizumab (8). A limited number of retrospective studies 
examined first-line chemotherapy for patients with dMMR CRCs. These studies indicated that, unlike patients whose tumours demonstrate pMMR, those with dMMR tumours did not benefit from 5-flurouracil/leucovorin $(9,10)$. In a hospital-based cohort study, patients with dMMR (18\%) and patients with pMMR colon cancers were found to benefit equally from 5-flurouracil/leucovorin chemotherapy (11). Conflicting data have been reported regarding dMMR $\mathrm{mCRCs}$ and 5-flurouracil/leucovorin response, and the examination of outcomes based on the presence of mutations in the MMR genes shows promise. Thus, our study's goal was to determine whether tumour MMR status was associated with improved response to IFL regimen in patients with mCRCs.

\section{Materials and Methods}

Study population. The study cohort was derived from the oncology and radiology department at Tuku University Hospital (TUH), Finland. Patients diagnosed with mCRC between August 1st, 1998, and August 30th, 2003, at TUH were included in this study. Patients were enrolled in the study if they were older than 18 years and diagnosed with a histologically confirmed right colon, transverse colon, left colon, or rectum cancer. Anatomic site of the tumour was determined by the treating surgeon. The study was approved by the National Authority for Medico-Legal Affairs Committee, Finland and was conducted in accordance with the declaration of Helsinki. Written informed consent was obtained from all patients included in this study.

Sample and immunostaining. A formalin-fixed, paraffin-embedded primary tumour was obtained from each patient. Sections were cut serially at $5 \mu \mathrm{m}$ for routine haematoxylin and eosin staining and for IHC analysis. An experienced pathologist confirmed all histological diagnoses. Evaluation and scoring of the IHC slides were carried out without knowledge of the clinical data.

Evaluation of hMLH1 and hMSH2 expression. Expression of hMLH1 and hMSH2 was studied using antibodies against hMLH1 and hMSH2 (Mouse anti-MLH1, Clone 14 and Mouse anti-MSH2, Clone FE11, Zymed Laboratories Inc., MA, USA) diluted in $1 \%$ bovine serum albumin/Tris-buffered saline $(1: 50$ or $1: 100$ respectively). Detection was performed using the streptavidin-biotin method (Vectastain ABC kit; Vector Laboratories, CA, USA). Two independent observers assessed the expression of hMLH1 and hMSH2. The slides were first screened for an overview of the general staining pattern. Loss of expression was recorded when nuclear staining was absent from cancer cell nuclei but preserved in normal epithelium and stroma. Tumour expression was classified as weak, moderate or strong relative to the expression of normal epithelial and stromal cells present in that tumour section.

Evaluation of VEGF-1 expression. For studying VEGF-1 expression, purified anti-human $\operatorname{VEGF}(121,165$, and 189 isoforms), clone VG-1 (Biosite company, Biosite, San Diego, CA, USA) (1:150), was used. After staining, the sections were dehydrated in ethanol, cleared in xylene and covered with Mountex and coverslips. The expression of VEGF-1 in the tumour tissue was assessed according to the expression in the total tumour area. Tumour tissue showed only cytoplasmic staining. The cytoplasmic staining was graded as: 0 ) negative, no detectable staining; 1 ) weak but detectable staining; 2) moderate, clearly positive staining; and 3) strong staining, intense throughout the tumour.

Evaluation of patient response to first-line IFL regimen. The study patients received a combination of irinotecan $\left(180-210 \mathrm{mg} / \mathrm{m}^{2}\right.$, administered as a 60-90 min intravenous infusion) and 5-fluorouracil (500 mg/m², i.v. bolus), modulated by leucovorin $\left(60 \mathrm{mg} / \mathrm{m}^{2}\right.$, i.v. bolus). The 5 -flurouracil/leucovorin administrations were repeated on the following day. This treatment combination was repeated every two weeks until disease progression or the occurrence of unacceptable toxicity. Tumour response was assessed every eight weeks by an independent reviewer according to WHO criteria $(12,13)$.

Statistical analysis. All analyses were performed with SPSS 21.0, and Sample Power 3.0 (SPSS). Categorical variables are presented herein as percentages, and continuous variables (age) are presented as means (SDs). The age variable was assessed for normality using the Kolmogorov-Smirnov test. The expression results of hMLH1 or $\mathrm{hMSH} 2$ were used as dichotomous variables, where tumours with negative or weak expression of either hMLH1 or hMSH2 were one category (MMR-deficient), and all those with moderate or strong expression were grouped into the second category (MMRproficient). The expression of VEGF-1 was also classified as a binary variable, where tumours with negative or weak staining were considered as having low VEGF-1 expression, and when tumours had moderate or strong staining were considered as having high VEGF-1 expression. Comparisons of categorical variables were performed using the chi-square $\left(\chi^{2}\right)$ test or Fisher's exact test. A finding of $p<0.05$ was considered indicative of a statistically significant difference for all tests.

\section{Results}

Tumour MMR- status and clinical characteristics. Baseline characteristics of the study cohort are shown in Table I. The mean age of patients was 58 years (range $=24-80$ years), and male sex was predominant consisting approximately twothirds $(64 \%)$ of the patients. Based on Tumour-NodeMetastasis (TNM) staging system, two-thirds of patients were diagnosed with T3 $(n=48,72 \%)$; half of the patients had at-least one node involvement $(n=35,52 \%)$; all study patients were diagnosed with mCRCs, and liver was the most common metastasis site $(n=28,42 \%)$. Of the 67 mCRCs patients, 29 (43\%) were hMLH1 or/and hMSH2-deficient, 15 $(22 \%)$ were proficient-MMR (pMMR), and 23 (35\%) were dMMR and pMMR (Table II).

Tumour MMR- status and VEGF-1 expression. At diagnosis, patients with hMLH1 or/and hMSH2-deficient tumours expressed lower levels of VEGF-1 as compared with pMMR tumour patients $(p=0.01)$ (Table I, Figure 1).

Tumour MMR-status and response to first-line IFL regimen. Among our study patients, more than half $(n=17,59 \%)$ of 
Table I. Baseline characteristics of the study cohort.

\begin{tabular}{|c|c|}
\hline Characteristics & $\begin{array}{c}\text { Total no. of patients } \\
67(\%)\end{array}$ \\
\hline \multicolumn{2}{|l|}{ Age } \\
\hline Mean (SD) & $57.6(24-80)$ \\
\hline \multicolumn{2}{|l|}{ Gender, no. (\%) } \\
\hline Female & $24(36)$ \\
\hline Male & $43(64)$ \\
\hline \multicolumn{2}{|c|}{ Location of the primary tumour, no. (\%) } \\
\hline Right colon & $15(22.4)$ \\
\hline Transverse colon & $7(10.4)$ \\
\hline Left colon & $31(46.3)$ \\
\hline Rectum & $14(20.9)$ \\
\hline \multicolumn{2}{|l|}{ TNM classification, no. $(\%)$} \\
\hline \multicolumn{2}{|l|}{ Tumour status } \\
\hline $\mathrm{T} 1$ & 0 \\
\hline $\mathrm{T} 2$ & $5(7.5)$ \\
\hline $\mathrm{T} 3$ & $48(71.6)$ \\
\hline $\mathrm{T} 4$ & $14(20.9)$ \\
\hline \multicolumn{2}{|l|}{ Nodal status } \\
\hline No & $18(26.9)$ \\
\hline N1 & $35(52.2)$ \\
\hline Unknown & $14(20.9)$ \\
\hline \multicolumn{2}{|l|}{$\begin{array}{l}\text { Location of metastases upon } \\
\text { starting chemotherapy, no. (\%) }\end{array}$} \\
\hline Local & $4(6)$ \\
\hline Liver & $28(41.8)$ \\
\hline Lung & $1(1.5)$ \\
\hline Multiple sites & $34(50.7)$ \\
\hline \multicolumn{2}{|l|}{ Histological grade, no. (\%) } \\
\hline Grade I & $10(14.9)$ \\
\hline Grade II & $45(67.2)$ \\
\hline Grade III & $12(17.9)$ \\
\hline \multicolumn{2}{|c|}{ MMR-status/ VEGF-1-staining, no. (\%) } \\
\hline dMMR/Weak & $29(43)$ \\
\hline pMMR/Strong & $15(23)$ \\
\hline Discordance cases/Strong & $23(34)$ \\
\hline
\end{tabular}

dMMR: Mismatch repair-deficient; pMMR: mismatch repair-proficient.

those with dMMR tumours $(n=29)$ were chemosensitive to first-line IFL regimen, while just one-fifth $(n=3,20 \%)$ of those with pMMR tumours $(\mathrm{n}=15)$ were chemosensitive to IFL regimen $(p=0.045)$ (Table II, Figure 2$)$.

\section{Discussion}

The present study provides insight into the role of MMR as a prognostic marker to guide treatment in mCRCs. This study analyzed data from 67 histopathologically-confirmed mCRC patients. There was a significant association between MMR-status and VEGF-1 expression. Compared to patients with dMMR tumours, patients with pMMR tumours expressed higher levels of VEGF-1 and had lower responserate to first-line IFL regimen.
Table II. Tumour MMR-status and response to first-line IFL regimen.

\begin{tabular}{lccc}
\hline MMR-status & $\begin{array}{c}\text { Total number } \\
\text { of patients } \\
67(\%)\end{array}$ & $\begin{array}{c}\text { Chemosensitive } \\
31(\%)\end{array}$ & $p$-Value \\
\hline dMMR tumours & 29 & $17(55)$ & 0.045 \\
pMMR tumours & 15 & $3(10)$ & \\
Discordance cases & 23 & $11(35)$ & \\
\hline
\end{tabular}

dMMR: Mismatch repair-deficient; pMMR: mismatch repair-proficient.

Our observation that dMMR tumours were associated with reduced levels of VEGF-1 compared to pMMR tumours, was in line with previous studies (14-17). Most of these studies, however, did not identify the relationship between MMR genes (i.e., MSH2, MLH1, MSH6, and PMS2) and VEGF-1 expression in dMMR mCRCs. One study found that there is a relationship between MLH1 and VEGF-1 expression in dMMR CRC patients (14). Among patients with an identified germline mutation in the MMR genes, mutations in MLH1, $M S H 2, M S H 6$, and $P M S 2$ are found to be in approximately $37,41,13$, and $9 \%$ of cases, respectively (17). A novel finding in our study was that we established a relationship between both hMSH2 and hMLH1, the most frequent reported mutant MMR genes, and VEGF-1 expression.

In this study, we found that compared to pMMR tumours, dMMR (hMSH2 and/or hHML1-defecient) tumours had higher response-rate to IFL regimen. Similarly, previous data in CRCs indicated that patients with dMMR tumours have a more favourable prognosis than those with pMMR tumours, and responded differently to 5-fluorouracil-based chemotherapy $(18,19)$. The reasonable explanation of the current results could be that patients with pMMR tumours expressed higher levels of VEGF-1 as compared with those with hMLH1 or/and hMSH2-deficient tumours. Several studies have reported the relationship between VEGF-1 status and the effect of the 5-fluorouracil-based chemotherapy $(20,21)$; patients expressing VEGF showed lower response-rate to chemotherapy, and had shorter overall survival than those who did not. The mechanism behind this relationship may be related to the pro-angiogenic effect of VEGF (22), which has a role in the progression of CRC (23). Increased VEGF levels is significantly associated with multiple lymph-nodes involvement and distance metastasis (24). Therefore, the expression of VEGF can be utilized as a predictive biomarker to drive personalized therapeutic decisions towards the addition of anti-VEGF, bevacizumab, to the 5-flurouracil-based chemotherapy regimen.

Among our study patients, more than half of the cohort with hMSH2 and/or hHML1-deficient tumours were responsive to the first-line IFL regimen, including 5flurouracil and leucovorin with irinotecan. This is consistent 

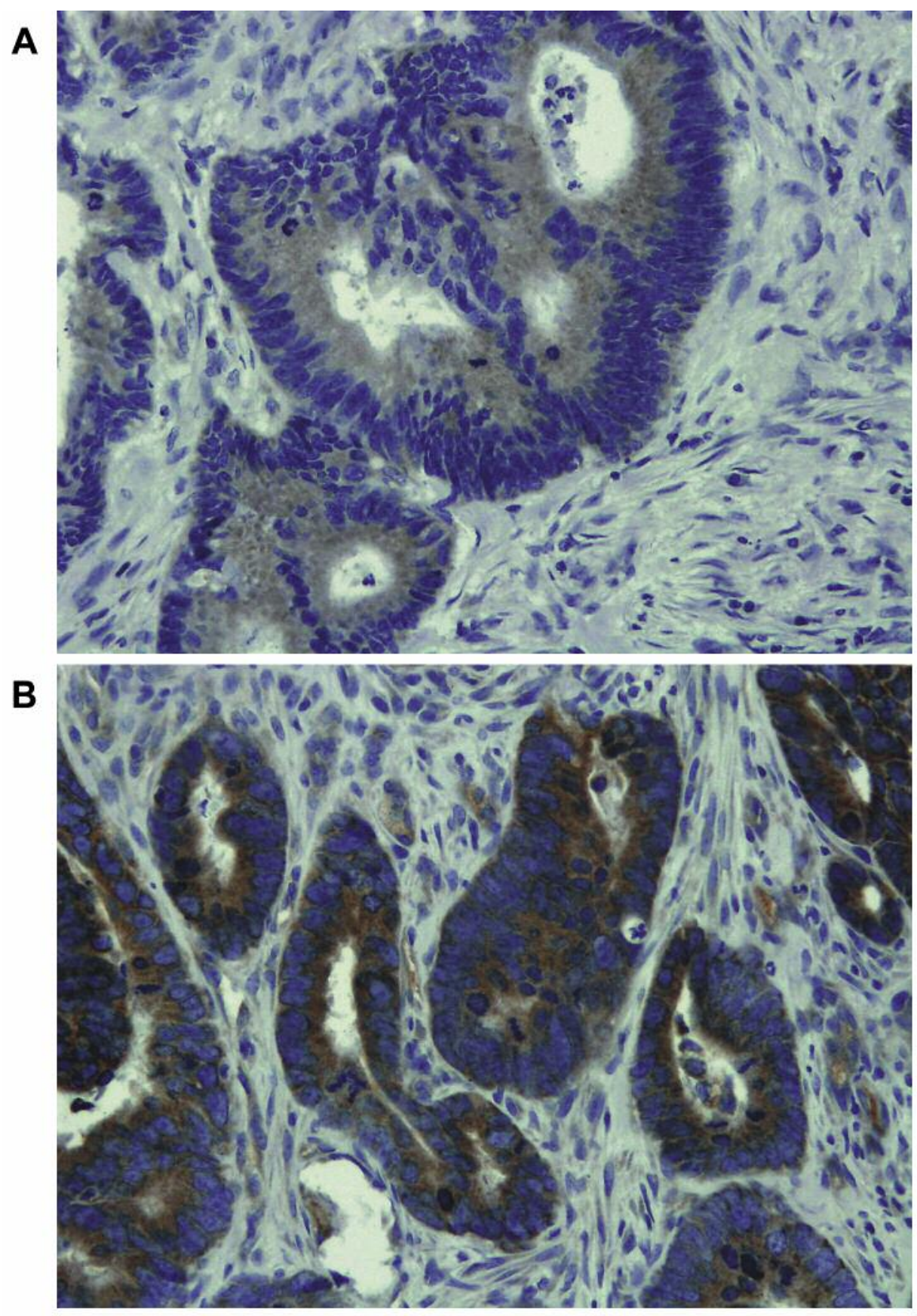

Figure 1. Representative images of VEGF-1 expression in colorectal cancer tissue. A: Weak cytoplasmic staining of VEGF-1 in MMR-deficient tumour. B: Strong cytoplasmic staining of VEGF-1 in MMR-proficient tumour.

with previous data showing that loss of tumour MMR proteins was associated with improved outcome in colon cancer patients treated with IFL regimen, as compared with those receiving standard 5-flurouracil/leucovorin chemotherapy (25). The possible mechanism underlying the responsiveness of dMMR tumours to IFL regimen, could be related to the irinotecan-induced cytotoxicity. In most tumours with suspected germline mutations, the dMMR phenotype results from the inactivation of either hMSH2 or hHML1, two components of MMR that regulate recombination. Irinotecan acts by generating double strand breaks in DNA, and, a decrease in recombinational repair 


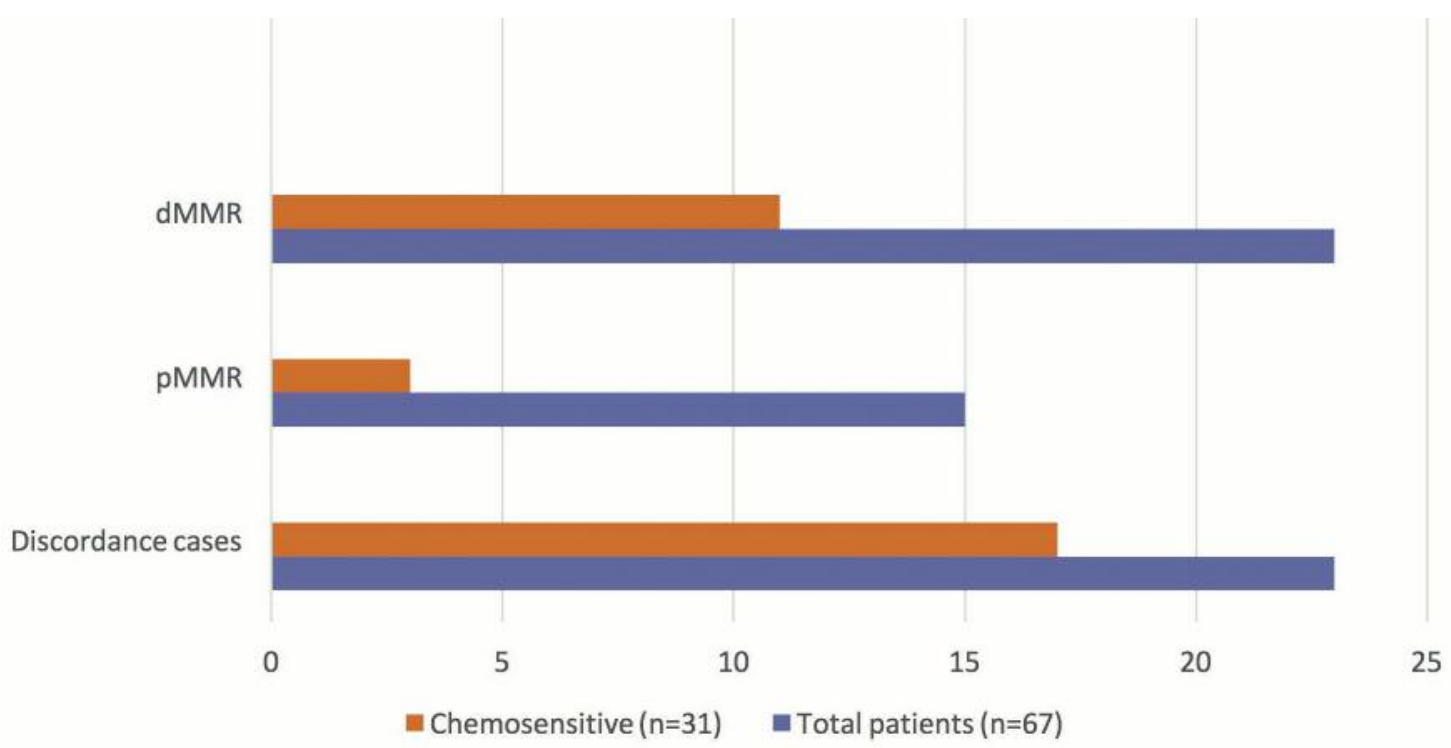

Figure 2. Response rate to first-line IFL regimen.

efficiency from loss of either hMSH2 or hHML-repair proteins could result in the higher sensitivity of dMMR tumours to chemotherapy (26).

Similar to any observational study, this investigation has a number of limitations. Although, our study was carried prospectively, there might be a number of unobservable factors that could only be controlled with a randomized controlled trial. In addition, the limited sample size of this study could have resulted in some bias in the results produced. Finally, we cannot totally exclude a non-MMR mechanism as being responsible for our findings. However, the benefits we have seen in patients with dMMR tumours treated with IFL suggest that the predictive value of tumour MMR status should be confirmed in larger randomized settings. Validation of our results will allow better selection of patients for chemotherapy.

In conclusion, pMMR tumours expressed higher levels of VEGF-1 and had lower response-rate to first-line IFL regimen. Therefore, a pMMR tumour subset could benefit from the addition of an anti-VEGF agent, such as bevacizumab. In the other hand, patients with hMLH1 or/and hMSH2-deficient tumours expressed lower levels of VEGF1 as compared with patients with pMMR tumours, and had higher response-rate to first-line 5-flurouracil/leucovorin with irinotecan chemotherapy. Future clinical trials in the mCRC settings should consider this molecular characteristic as an important stratification factor.

\section{Conflicts of Interest}

There are no conflicts of interest regarding this study.

\section{References}

1 Torre LA, Bray F, Siegel RL, Ferlay J, Lortet-Tieulent J and Jemal A: Global cancer statistics, 2012. CA Cancer J Clin 65(2): 87-108, 2015.

2 Lengauer C, Kinzler KW and Vogelstein B: Genetic instability in colorectal cancers. Nature 386(6625): 623, 1997.

3 Kawakami H, Zaanan A and Sinicrope FA: Implications of mismatch repair-deficient status on management of early stage colorectal cancer. J Gastrointest Oncol 6(6): 676, 2015.

4 Shia J, Ellis NA and Klimstra DS: The utility of immunohistochemical detection of DNA mismatch repair gene proteins. Virchows Arch 445(5): 431-441, 2004.

5 Douillard J, Cunningham D, Roth AD, Navarro M, James RD, Karasek P, Jandik P, Iveson T, Carmichael J, Alakl M and Gruia G: Irinotecan combined with fluorouracil compared with fluorouracil alone as first-line treatment for metastatic colorectal cancer: a multicentre randomised trial. The Lancet 355(9209): 1041-1047, 2000.

6 Saltz LB, Cox JV, Blanke C, Rosen LS, Fehrenbacher L, Moore MJ, Maroun JA, Ackland SP, Locker PK, Pirotta N and Elfring GL: Irinotecan plus fluorouracil and leucovorin for metastatic colorectal cancer. N Engl J Med 343(13): 905-914, 2000.

7 Goldberg RM, Sargent DJ, Morton RF, Fuchs CS, Ramanathan RK, Williamson SK, Findlay BP, Pitot HC and Alberts SR: A randomized controlled trial of fluorouracil plus leucovorin, irinotecan, and oxaliplatin combinations in patients with previously untreated metastatic colorectal cancer. J Clin Oncol 22(1): 23-30, 2004.

8 Van Cutsem $\mathrm{E}$ and Geboes $\mathrm{K}$ : The integration of cytotoxics and biologicals in the treatment of metastatic colorectal cancer. Best Pract Res Clin Gastroenterol 21(6): 1089-1108, 2007.

9 Ribic CM, Sargent DJ, Moore MJ, Thibodeau SN, French AJ, Goldberg RM, Hamilton SR, Laurent-Puig P, Gryfe R, Shepherd LE and Tu D: Tumor microsatellite-instability status as a predictor of benefit from fluorouracil-based adjuvant chemotherapy for colon cancer. N Engl J Med 349(3): 247-257, 2003. 
10 Kim GP, Colangelo LH, Wieand HS, Paik S, Kirsch IR, Wolmark N and Allegra CJ: Prognostic and predictive roles of high-degree microsatellite instability in colon cancer: a National Cancer Institute-National Surgical Adjuvant Breast and Bowel Project Collaborative Study. J Clin Oncol 25(7): 767-772, 2007.

11 Allegra CJ, Paik S, Colangelo LH, Parr AL, Kirsch I, Kim G, Klein P, Johnston PG, Wolmark N and Wieand HS: Prognostic value of thymidylate synthase, $\mathrm{Ki}-67$, and p53 in patients with Dukes' B and C colon cancer: A national cancer institutenational surgical adjuvant breast and bowel project collaborative study. J Clin Oncol 21(2): 241-250, 2003.

12 Miller AB, Hoogstraten BF, Staquet MF and Winkler A: Reporting results of cancer treatment. Cancer 47(1): 207-214, 1981.

13 Therasse P, Arbuck SG, Eisenhauer EA, Wanders J, Kaplan RS, Rubinstein L, Verweij J, Van Glabbeke M, Van Oosterom AT, Christian MC and Gwyther SG: New guidelines to evaluate the response to treatment in solid tumors. J Natl Cancer Inst 92(3): 205-216, 2000.

14 Ricciardiello L, Ceccarelli C, Angiolini G, Pariali M, Chieco P, Paterini P, Biasco G, Martinelli GN, Roda E and Bazzoli F: High thymidylate synthase expression in colorectal cancer with microsatellite instability: implications for chemotherapeutic strategies. Clin Cancer Res 11(11): 4234-4240, 2005.

15 Wendum D, Comperat E, Boëlle PY, Parc R, Masliah J, Trugnan $\mathrm{G}$ and Fléjou JF: Cytoplasmic phospholipase A2 alpha overexpression in stromal cells is correlated with angiogenesis in human colorectal cancer. Mod Pathol 18(2): 212, 2005.

16 Zhang CM, Lv JF, Gong L, Yu Yu, Chen XP, Zhou HH and Fan L: Role of deficient mismatch repair in the personalized management of colorectal cancer. Int $\mathrm{J}$ Environ Res Public Health 13(9): 892, 2016.

17 Moreira L, Balaguer F, Lindor N, De La Chapelle A, Hampel H, Aaltonen LA, Hopper JL, Le Marchand L, Gallinger S, Newcomb PA and Haile R: Identification of Lynch syndrome among patients with colorectal cancer. JAMA 308(15): 1555-1565, 2012.

18 Lanza G, Gafà R, Santini A, Maestri I, Guerzoni L and Cavazzini L: Immunohistochemical test for MLH1 and MSH2 expression predicts clinical outcome in stage II and III colorectal cancer patients. J Clin Oncol 24(15): 2359-2367, 2006.

19 Elsaleh H, Joseph D, Grieu F, Zeps N, Spry N and Iacopetta B: Association of tumour site and sex with survival benefit from adjuvant chemotherapy in colorectal cancer. The Lancet 355(9217): 1745-1750, 2000.
20 Hirashima Y, Yamada Y, Matsubara J, Takahari D, Okita N, Takashima A, Kato K, Hamaguchi T, Shirao K, Shimada Y and Taniguchi H: Impact of vascular endothelial growth factor receptor 1,2, and 3 expression on the outcome of patients with gastric cancer. Cancer Sci 100(2): 310-315, 2009.

21 Bendardaf R, Buhmeida A, Hilska M, Laato M, Syrjänen S, Syrjänen K, Collan Y and Pyrhönen S: VEGF-1 expression in colorectal cancer is associated with disease localization, stage, and long-term disease-specific survival. Anticancer Res 28(6B): 3865-3870, 2008.

22 Eichhorn ME, Kleespies A, Angele MK, Jauch KW and Bruns CJ: Angiogenesis in cancer: molecular mechanisms, clinical impact. Langenbecks Arch Surg 392(3): 371-379, 2007.

23 Guba M, Seeliger H, Kleespies A, Jauch KW and Bruns CJ: Vascular endothelial growth factor in colorectal cancer. Int J Colorectal Dis 19(6): 510-517, 2004.

24 Stacker SA, Williams SP, Karnezis T, Shayan R, Fox SB and Achen MG: Lymphangiogenesis and lymphatic vessel remodelling in cancer. Nat Rev Cancer 14(3): 159, 2014.

25 Bertagnolli MM, Niedzwiecki D, Compton CC, Hahn HP, Hall M, Damas B, Jewell SD, Mayer RJ, Goldberg RM, Saltz LB, Warren RS and Redston M: Microsatellite instability predicts improved response to adjuvant therapy with irinotecan, fluorouracil, and leucovorin in stage III colon cancer: Cancer and Leukemia Group B Protocol 89803. J Clin Oncol 27(11): 1814-1821, 2009.

26 Fallik D, Borrini F, Boige V, Viguier J, Jacob S, Miquel C, Sabourin JC, Ducreux M and Praz F: Microsatellite instability is a predictive factor of the tumor response to irinotecan in patients with advanced colorectal cancer. Cancer Res 63(18): 5738-5744, 2003. 